\title{
Approximating the Solution of the Linear and Nonlinear Fuzzy Volterra Integrodifferential Equations Using Expansion Method
}

\author{
T. Allahviranloo, S. Abbasbandy, and S. Hashemzehi \\ Department of Mathematics, Science and Research Branch, Islamic Azad University, Tehran, Iran \\ Correspondence should be addressed to T. Allahviranloo; tofigh@allahviranloo.com
}

Received 26 January 2014; Accepted 19 February 2014; Published 6 April 2014

Academic Editor: Reza Ezzati

Copyright ( 2014 T. Allahviranloo et al. This is an open access article distributed under the Creative Commons Attribution License, which permits unrestricted use, distribution, and reproduction in any medium, provided the original work is properly cited.

The present research study introduces an innovative method applying power series to solve numerically the linear and nonlinear fuzzy integrodifferential equation systems. Finally, it ends with some examples supporting the idea.

\section{Introduction}

Fuzzy integrodifferential equations have attracted great interests in recent years since they play a major role in different areas of theory such as control theory. For the first time, Chang and Zadeh have introduced fuzzy numbers as well as the related arithmetic operations $[1,2]$. Furthermore, applying the operators on fuzzy numbers has been developed by Mizumoto and Tanaka [3]. It should be mentioned that the concept of LR fuzzy numbers was expressed by Dubois and Prade [4]. In this regard, they made a significant contribution by providing a computational formula for operations on fuzzy numbers. After that, the notation of fuzzy derivative was presented by Seikkala [5]. However, Goetschel, Jr., and Voxman have proposed the Riemann integral-type approach [6]. Some mathematicians have separately worked on the existence and having a unique solution of fuzzy Volterra integrodifferential equation [7-9]. Recently, numerical methods have been applied to solve the linear as well as nonlinear differential equation fuzzy integral equation and fuzzy integrodifferential equation $[7,8,10-13]$.

In this paper, we use the power series method of the exact solution of linear or nonlinear fuzzy integrodifferential equations, which is obtained by recursive procedure as follows.

We consider the following system of fuzzy integrodifferential equations:

$$
\widetilde{X}^{\prime}(s)=G(s, \widetilde{X}(s)) \oplus \int_{0}^{s} k\left(s, t, \widetilde{X}(t), \widetilde{X}^{\prime}(t)\right) d t,
$$

with initial condition $\widetilde{F}(0)=\widetilde{a}$, and

$$
\begin{aligned}
\widetilde{X} & =\left[\widetilde{x}_{1}, \widetilde{x}_{2}, \ldots, \widetilde{x}_{n}\right]^{T}, \\
\widetilde{G} & =\left[\widetilde{g}_{1}, \widetilde{g}_{2}, \ldots, \widetilde{g}_{n}\right]^{T}, \\
\widetilde{K} & =\left[\widetilde{k}_{i j}\right], \quad i, j=1,2, \ldots, n, \\
\widetilde{a} & =\left[\widetilde{a}_{1}, \widetilde{a}_{2}, \ldots, \widetilde{a}_{n}\right]^{T} .
\end{aligned}
$$

In (1), $\widetilde{G}$ and $\widetilde{K}$ are given fuzzy functions and, also, $\widetilde{a}$ is fuzzy vector and vector fuzzy function $\widetilde{X}$ is solution of (1), which will be determined.

\section{Basic Concepts}

Here basic definitions of a fuzzy number are given as follows [14-19].

Let $E$ be a set of all triangular fuzzy numbers.

Definition 1. An arbitrary fuzzy number $\widetilde{u} \in E$ in the parametric form is represented by an ordered pair of functions $(\underline{u}, \bar{u})$ which satisfy the following requirements.

(i) $\bar{u}: r \rightarrow \underline{u}(r) \in \mathbb{R}$ is a bounded left-continuous nondecreasing function over $[0,1]$.

(ii) $\underline{u}: r \rightarrow \bar{u}(r) \in \mathbb{R}$ is a bounded left-continuous nonincreasing function over $[0,1]$.

(iii) $u \leq \bar{u}, \quad 0 \leq r \leq 1$. 
Definition 2. For arbitrary fuzzy numbers $\tilde{u}, \widetilde{v} \in E$, one uses the distance (Hausdorff metric) [6]

$$
\begin{array}{r}
D(u(r), v(r))=\max \left\{\sup _{r \in[0,1]}|\underline{u}(r)-\underline{v}(r)|,\right. \\
\left.\sup _{r \in[0,1]}|\bar{u}(r)-\bar{v}(r)|\right\},
\end{array}
$$

and it is shown in [6] that $(E, D)$ is a complete metric space and the following properties are well known:

$$
\begin{gathered}
D(\widetilde{u}+\widetilde{w}, \widetilde{v}+\widetilde{w})=D(\widetilde{u}, \widetilde{v}), \quad \forall \widetilde{u}, \widetilde{v} \in E, \\
D(k \widetilde{u}, k \widetilde{v})=|k| D(\widetilde{u}, \widetilde{v}), \quad \forall k \in \mathbb{R}, \widetilde{u}, \widetilde{v} \in E, \\
D(\widetilde{u}+\widetilde{v}, \widetilde{w}+\widetilde{e}) \leq D(\widetilde{u}, \widetilde{w})+D(\widetilde{v}, \widetilde{e}), \quad \forall \widetilde{u}, \widetilde{v}, \widetilde{w}, \widetilde{e} \in E .
\end{gathered}
$$

Definition 3. A triangular fuzzy number is defined as a fuzzy set in $E$, which is specified by an ordered triple $u=(a, b, c) \in$ $\mathbb{R}^{3}$ with $a \leq b \leq c$ such that $[u]^{r}=[\underline{u}(r), \bar{u}(r)]$ are the endpoints of $r$-level sets for all $r \in[0,1]$, where $\underline{u}(r)=$ $a+(b-a) r$ and $\bar{u}(r)=c-(c-b) r$. Here, $\underline{u}(0)=a, \bar{u}(0)=$ $c, \underline{u}(1)=\bar{u}(1)=b$, which is denoted by $u^{1}$.

Definition 4. A fuzzy number $\widetilde{A}$ is of $L R$ type if there exist shape functions $L$ (for left), $R$ (for right), and scalar $\alpha \geq$ $0, \beta \geq 0$ with

$$
\tilde{\mu}_{A}(x)= \begin{cases}L\left(\frac{a-x}{\alpha}\right) & x \leq a, \\ R\left(\frac{x-a}{\beta}\right) & x \geq a ;\end{cases}
$$

the mean value of $\widetilde{A}, a$ is a real number, and $\alpha, \beta$ are called the left and right spreads, respectively. $\widetilde{A}$ is denoted by $(a, \alpha, \beta)$.

Definition 5. Let $\widetilde{M}=(m, \alpha, \beta)_{L R}, \widetilde{N}=(n, \gamma, \delta)_{L R}$, and $\lambda \epsilon$ $\mathbb{R}^{+}$. Then,

$$
\begin{aligned}
\text { (1) } \lambda \widetilde{M} & =(\lambda m, \lambda \alpha, \lambda \beta)_{L R} \\
\text { (2) } \lambda \widetilde{M} & =(-\lambda m, \lambda \beta, \lambda \alpha)_{L R} \\
\text { (3) } \quad \widetilde{M} \oplus \widetilde{N}=(m+n, \alpha+\gamma, \beta+\delta)_{L R} & \\
\text { (4) } \quad \widetilde{M} \odot \widetilde{N} & \simeq \begin{cases}(m n, m \gamma+n \alpha, m \delta+n \beta)_{L R} & \widetilde{M}, \widetilde{N}>0 \\
(m n, n \alpha-m \delta, n \beta-m \gamma)_{L R} & \widetilde{M}>0, \widetilde{N}<0 \\
(m n,-n \beta-m \delta,-n \alpha-m \gamma)_{L R} & \widetilde{M}, \widetilde{N}<0 .\end{cases}
\end{aligned}
$$

Definition 6. The integral of a fuzzy function was defined in [6] by using the Riemann integral concept.
Let $f:[a, b] \rightarrow E^{1}$, for each partition $P=\left\{t_{0}, t_{1}, \ldots, t_{n}\right\}$ of $[a, b]$ and for arbitrary $\xi_{i} \in\left[t_{i}-1, t_{i}\right], 1 \leq i \leq n$, and suppose

$$
\begin{gathered}
R_{p}=\sum_{i=1}^{n} f\left(\xi_{i}\right)\left(t_{i}-t_{i-1}\right), \\
\Delta:=\max \left\{\left|t_{i}-t_{i-1}\right|, 1 \leq i \leq n\right\} .
\end{gathered}
$$

The definite integral of $f(t)$ over $[a, b]$ is

$$
\int_{a}^{b} f(t) d t=\lim _{\Delta \rightarrow 0} R_{p}
$$

provided that this limit exists in the metric $D$.

If the fuzzy function $f(t)$ is continuous in the metric $D$, its definite integral exists [17], and also

$$
\begin{aligned}
& \left(\underline{\left.\int_{a}^{b} f(t, r) d t\right)}=\int_{a}^{b} \underline{f}(t, r) d t\right. \\
& \left(\overline{\left.\int_{a}^{b} f(t, r) d t\right)}=\int_{a}^{b} \bar{f}(t, r) d t .\right.
\end{aligned}
$$

Definition 7 (see [14]). Consider $\tilde{x}, \tilde{y} \in E$. If there exists $\widetilde{z} \in E$ such that $\tilde{x}=\tilde{y}+\widetilde{z}$, then $\widetilde{z}$ is called the H-difference of $\tilde{x}$ and $\tilde{y}$ and is denoted by $\tilde{x} \ominus \tilde{y}$.

Proposition 8 (see [14]). If $\tilde{f}:(a, b) \rightarrow E$ is a continuous fuzzy-valued function, then $\tilde{g}(x)=\int_{a}^{x} \widetilde{f}(t)$ is differentiable, with derivative $\tilde{g}^{\prime}(x)=\widetilde{f}(x)$.

Definition 9 (see [20]). Let $f: R \rightarrow E$ be a fuzzy valued function. If, for arbitrary fixed $t_{0} \in R$ and $\epsilon>0$, a $\delta>0$ such that

$$
\left|t-t_{0}\right|<\delta \Longrightarrow d\left(f(t), f\left(t_{0}\right)\right)<\epsilon,
$$

$f$ is said to be continuous.

Definition 10 (see [21]). Let be $\tilde{f}:(a, b) \rightarrow E$ and $x_{0} \in(a, b)$. One says that $\tilde{f}$ is differentiable at $x_{0}$ if

(1) there exists an element $\tilde{f}^{\prime}\left(x_{0}\right) \in E$ such that, for all $h>0$ sufficiently near to 0 , there are $\tilde{f}\left(x_{0}+h\right) \ominus$ $\widetilde{f}\left(x_{0}\right), \exists \widetilde{f}\left(x_{0}\right) \ominus \widetilde{f}\left(x_{0}-h\right)$, and the limits

$$
\begin{aligned}
\lim _{h \rightarrow 0^{+}} & \frac{\tilde{f}\left(x_{0}+h\right) \ominus \tilde{f}\left(x_{0}\right)}{h} \\
\quad & \lim _{h \rightarrow 0^{+}} \frac{\tilde{f}\left(x_{0}\right) \ominus \tilde{f}\left(x_{0}-h\right)}{h}=\tilde{f}^{\prime}\left(x_{0}\right)
\end{aligned}
$$


(2) there exists an element $\tilde{f}^{\prime}\left(x_{0}\right) \in E$ such that, for all $h<$ 0 , sufficiently near to 0 , there are $\widetilde{f}\left(x_{0}+h\right) \ominus \widetilde{f}\left(x_{0}\right), \exists \widetilde{f}\left(x_{0}\right) \ominus$ $\widetilde{f}\left(x_{0}-h\right)$, and the limits

$$
\begin{aligned}
\lim _{h \rightarrow 0^{-}} & \frac{\tilde{f}\left(x_{0}+h\right) \ominus \tilde{f}\left(x_{0}\right)}{h} \\
\quad & =\lim _{h \rightarrow 0^{-}} \frac{\tilde{f}\left(x_{0}\right) \ominus \tilde{f}\left(x_{0}-h\right)}{h}=\tilde{f}^{\prime}\left(x_{0}\right) .
\end{aligned}
$$

Lemma 11 (see [14]). For $\tilde{x}_{0} \in R$ the fuzzy differential equation

$$
\begin{aligned}
\tilde{y}^{\prime} & =\tilde{f}(x, y), \\
\tilde{y}\left(x_{0}\right) & =\tilde{y}_{0} \in E,
\end{aligned}
$$

where $\tilde{f}: R \times E \rightarrow E$ is supposed to be continuous, if equivalent to one of the integral equations:

$$
\tilde{y}(x)=\tilde{y}_{0}+\int_{x_{0}}^{x} f(t, \tilde{y}(t)), \quad \forall x \in\left[x_{0}, x_{1}\right]
$$

or

$$
\tilde{y}(x)=\tilde{y}_{0}+(-1) \int_{x_{0}}^{x} f(t, \tilde{y}(t)), \quad \forall x \in\left[x_{0}, x_{1}\right] .
$$

On some interval $\left(x_{0}, x_{1}\right)$ under the differentiability condition, (i) or (ii), respectively.

Definition 12 (see [22]). For fuzzy number $\widetilde{u}(r)=$ $(\underline{u}(r), \bar{u}(r)), 0 \leq r \leq 1$, one writes (1) $\widetilde{u}>0$, if $\underline{u}(r)>0$, (2) $\widetilde{u} \geq 0$, if $\underline{u}(r) \geq 0$, (3) $\widetilde{u}<0$, if $\bar{u}<0$, and (4) $\widetilde{u} \leq 0$, if $\bar{u} \leq 0$.

Theorem 13 (see [14]). Let $c \in E$ and $g:(a, b) \rightarrow R$. If $g$ is differentiable on $x_{0}$, then the function $f:(a, b) \rightarrow R$, defined by $f(x)=c \odot g(x), \forall x \in(a, b)$, is differentiable on $x_{0}$ and one has $f^{\prime}\left(x_{0}\right)=c \odot g^{\prime}\left(x_{0}\right)$.

Corollary 14 (see [14]). Let $c \in E$ and $g:(a, b) \rightarrow R$. And define $f:(a, b) \rightarrow R$ by $f(x)=c \odot g(x), \forall x \in(a, b)$. If $g$ is differentiable on $(a, b)$ and $g^{\prime}$ is differentiable on $x_{0} \in(a, b)$, then $f$ is differentiable on $(a, b)$ and twice differentiable on $x_{0}$, with $f^{\prime \prime}\left(x_{0}\right)=c \odot g^{\prime \prime}\left(x_{0}\right)$.

Remark 15 (see [14]). In general, if the above $g$ is $n-1$ times differentiable on $(a, b)$ and $g^{n-1}$ is differentiable on $x_{0}$, then $f(x)=c \odot g(x)$ is differentiable of order $n$ on $x_{0}$ and $f^{n}\left(x_{0}\right)=$ $c \odot g^{n}\left(x_{0}\right)$.

Theorem 16 (see [21]). Let $\tilde{f}:(a, b) \rightarrow$ E be a function and denote $[\widetilde{f}(x)]^{r}=[f(r), \bar{f}(r)]$, for each $r \in[0,1]$. Then one has the following.

(i) If $\tilde{f}$ is differentiable in the first form (Definition 10), then $\underline{f}, \bar{f}$ are differentiable functions and

$$
\left[\tilde{f}^{\prime}(x)\right]^{r}=\left[\underline{f}^{\prime}(x), \bar{f}^{\prime}(x)\right] .
$$

(ii) If $\tilde{f}$ is differentiable in the second form (Definition 10), then $f, \bar{f}$ are differentiable functions and

$$
\left[\tilde{f}^{\prime}(x)\right]^{r}=\left[\bar{f}^{\prime}(x), \underline{f}^{\prime}(x)\right] .
$$

\section{Approximation Based on the Expansion Method}

Since $s$ is positive so all derivatives of $\widetilde{X}(s)$ in (18) are in case (i) in Definition 10.

Suppose the solution of the system of fuzzy integrodifferential equations (1) is as follows:

$$
\widetilde{X}_{i}(s)=\sum_{j=0}^{m} \widetilde{e}_{i j} s^{j}, \quad i=1,2, \ldots, n,
$$

where $\widetilde{e}_{i j} \in E$, for all $i=1,2, \ldots, n$. By using initial conditions, we have

$$
\widetilde{e}_{i 0}=\widetilde{x}_{i}(0), \quad i=1,2, \ldots, n .
$$

The coefficients of (18) are computed step by step. Firstly, the solution of problem (1) is considered as

$$
\widetilde{X}(s)=\widetilde{e}_{0} \oplus \widetilde{e}_{1} \odot s,
$$

where $\widetilde{e}_{i j}, i=1,2, \ldots, n$, and $\widetilde{e}_{1}$ are unknown. With derivative of (20) we have $\widetilde{X}^{\prime}(s)=\widetilde{e}_{1}$ and by substituting $\widetilde{X}^{\prime}(s),(20)$ into (1), we have

$$
\widetilde{e}_{1}=G\left(s, \widetilde{e}_{0} \oplus \widetilde{e}_{1} \odot s\right) \oplus \int_{0}^{s} k\left(s, t, \widetilde{e}_{0} \oplus \widetilde{e}_{1} \odot s, \widetilde{e}_{1}\right) d t,
$$

where by integration and sort of terms of above equation we obtain the following system:

$$
\left(A_{1} \odot \widetilde{e}_{1} \ominus \widetilde{b}_{1}\right) \oplus \widetilde{Q}_{1}(s)=\widetilde{0},
$$

where $A_{1}$ is $n \times n$ constant matrix, $\widetilde{b}_{1}$ is $n \times 1$ fuzzy vector, $\widetilde{Q}_{1}(s)=\left[\widetilde{q}_{i 1}(s)\right], i=1,2, \ldots, n$, and $\widetilde{q}_{i 1}(s)$ are polynomials of order equal or greater than 1 . If $s=0$ by neglecting $\widetilde{Q}_{1}(s)$, we have fuzzy linear equations system of $\widetilde{e}_{1}$. By solving this system, the coefficient of $\widetilde{x}$ in (20) can be determined.

In the second step, we assume that

$$
\widetilde{X}(s)=\widetilde{e}_{0} \oplus \widetilde{e}_{1} \odot s \oplus \widetilde{e}_{2} \odot s^{2},
$$

where $\widetilde{e}_{0}$ and $\widetilde{e}_{1}$ are known and $\widetilde{e}_{2}$ is unknown. With derivative of (23) we have

$$
\begin{aligned}
& \text { case (1), if } \widetilde{e}_{1}>0: \widetilde{X}^{\prime}(s)=\widetilde{e}_{1} \oplus 2 \widetilde{e}_{2} \odot s ; \\
& \text { case (2), if } \widetilde{e}_{1}<0: \widetilde{X}^{\prime}(s)=\ominus \widetilde{e}_{1} \oplus 2 \widetilde{e}_{2} \odot s,
\end{aligned}
$$

and by substituting $\widetilde{X}^{\prime}(s),(23)$ into (1), we have

$$
\begin{array}{r}
\widetilde{e}_{1} \oplus 2 \widetilde{e}_{2} \odot s=G\left(s, \widetilde{e}_{0} \oplus \widetilde{e}_{1} \odot s \oplus \widetilde{e}_{2} \odot s^{2}\right) \\
\oplus \int_{0}^{s} k\left(s, t, \widetilde{e}_{0} \oplus \widetilde{e}_{1} \odot s \oplus \widetilde{e}_{2}\right. \\
\left.\odot s^{2}, \widetilde{e}_{1} \oplus 2 \widetilde{e}_{2} \odot s\right) d t
\end{array}
$$


or

$$
\begin{array}{r}
\ominus \widetilde{e}_{1} \oplus 2 \widetilde{e}_{2} \odot s=G\left(s, \widetilde{e}_{0} \oplus \widetilde{e}_{1} \odot s \oplus \widetilde{e}_{2} \odot s^{2}\right) \\
\oplus \int_{0}^{s} k\left(s, t, \widetilde{e}_{0} \oplus \widetilde{e}_{1} \odot s \oplus \widetilde{e}_{2}\right. \\
\left.\odot s^{2}, \ominus \widetilde{e}_{1} \oplus 2 \widetilde{e}_{2} \odot s\right) d t
\end{array}
$$

where by integration and sort of terms of above equation we obtain the following system:

$$
\left(A_{2} \odot \widetilde{e}_{2} \ominus \widetilde{b}_{2}\right) \oplus \widetilde{Q}_{2}(s)=\widetilde{0},
$$

where $A_{2}$ is $n \times n$ constant matrix, $\widetilde{b}_{2}$ is $n \times 1$ fuzzy vector $A_{1}$, and $\widetilde{Q}_{2}(s)=\left[\widetilde{q}_{i 2}(s)\right], i=1,2, \ldots, n$, and $\tilde{q}_{i 2}(s)$ are polynomials of order greater than unity, where by neglecting $\widetilde{Q}_{2}(s)$, we have again fuzzy system of linear equations of $\widetilde{e}_{2}$ and by solving this system, coefficients of $s^{2}$ in (23) can be determined. This procedure can be repeated till the arbitrary order coefficients of power series of the solution for the problem are obtained.

The following theorem shows convergence of the method. Without loss of generality, we prove it for $n=1$.

Theorem 17. Let $\widetilde{X}=\widetilde{F}(s)$ be the exact solution of the following fuzzy integrodifferential equation:

$$
\begin{gathered}
\widetilde{X}^{\prime}(s)=G(s, \widetilde{X}(s)) \oplus \int_{0}^{s} k\left(s, t, \widetilde{X}(t), \widetilde{X}^{\prime}(t)\right) d t \\
\widetilde{X}(0)=\widetilde{a} .
\end{gathered}
$$

Assume that $\tilde{f}(s)$ has a power series representation. Then,

$$
\lim _{m \rightarrow \infty} \widetilde{f}_{m}(s)=\widetilde{f}(s) .
$$

Proof. According to the proposed method, we assume that the approximate solution to (27) is as follows:

$$
\tilde{f}_{m}(s)=\widetilde{e}_{0} \oplus \tilde{e}_{1} \odot s \oplus \tilde{e}_{2} \odot s^{2} \oplus \cdots \oplus \tilde{e}_{m} \odot s^{m} .
$$

Hence, it is sufficient that we only prove

$$
\begin{array}{r}
\text { if: } \widetilde{e}_{m}>0, \quad \widetilde{e}_{m}=\frac{\widetilde{f}^{(m)}(0)}{m !}, \\
\text { if: } \widetilde{e}_{m}<0, \quad \ominus \widetilde{e}_{m}=\frac{\widetilde{f}^{(m)}(0)}{m !}
\end{array}
$$

for $m=1,2,3, \ldots$.

Note that, for $m=0$, the initial condition gives

$$
\widetilde{e}_{0}=\tilde{f}(0)=\widetilde{a}
$$

Moreover, for $m=1$, if we set $\widetilde{X}=\widetilde{f}(s)$ and $s=0$ in (27), we obtain

$$
\widetilde{f}^{\prime}(0)=g(0, \widetilde{f}(0)) \oplus \widetilde{0}
$$

On the other hand, from (29) and (31), we have

$$
\tilde{f}_{1}(s)=\widetilde{e}_{0} \oplus \widetilde{e}_{1} \odot s .
$$

By substituting (33) into (27) and setting $s=0$, we get

$$
\begin{array}{r}
\text { if: } \widetilde{e}_{1}>0, \quad \widetilde{e}_{1}=g(0, \tilde{f}(0)) \oplus \widetilde{0}=\widetilde{f}^{\prime}(0), \\
\text { if: } \widetilde{e}_{1}<0, \quad \ominus \widetilde{e}_{1}=g(0, \tilde{f}(0)) \oplus \widetilde{0}=\widetilde{f}^{\prime}(0) .
\end{array}
$$

For $m=2$, differentiating (27) with respect to $s$, we have

$$
\begin{aligned}
\tilde{f}^{\prime \prime}(s)= & \frac{\sigma}{\sigma s} g(s, \tilde{f}(s)) \oplus \frac{\sigma}{\sigma s} g(s, \widetilde{f}(s)) \tilde{f}^{\prime}(s) \\
& \oplus K\left(s, \tilde{f}(s), \widetilde{f}^{\prime}(s)\right) \\
& \oplus \int_{0}^{s} \frac{\sigma}{\sigma s} K\left(s, t, \tilde{f}(t), \tilde{f}^{\prime}(t)\right) d t
\end{aligned}
$$

Setting $s=0$ in (35), we get

$$
\begin{aligned}
\tilde{f}^{\prime \prime}(0)=\frac{\sigma}{\sigma s} g(0, \tilde{f}(0)) & \oplus \frac{\sigma}{\sigma s} g(0, \tilde{f}(0)) \tilde{f}^{\prime}(0) \\
& \oplus K\left(0, \tilde{f}(0), \widetilde{f}^{\prime}(0)\right) .
\end{aligned}
$$

According to (29), (31), and (34), let

$$
\widetilde{f}_{2}(s)=\tilde{f}(0) \oplus \widetilde{f}^{\prime}(0) \odot s \oplus \widetilde{e}_{2} \odot s^{2} .
$$

By substituting (37) into (35) and setting $s=0$, we obtain

if: $\widetilde{e}_{2}>0, \widetilde{e}_{1}>0$,

$$
2 \widetilde{e}_{2}=\frac{\sigma}{\sigma s} g\left(0, \widetilde{e}_{0}\right) \oplus \frac{\sigma}{\sigma s} g\left(0, \widetilde{e}_{0}\right) \widetilde{e}_{1} \oplus K\left(0, \widetilde{e}_{0}, \widetilde{e}_{1}\right)
$$

if: $\tilde{e}_{2}>0, \tilde{e}_{1}<0$,

$$
2 \widetilde{e}_{2}=\frac{\sigma}{\sigma s} g\left(0, \widetilde{e}_{0}\right) \oplus \frac{\sigma}{\sigma s} g\left(0, \widetilde{e}_{0}\right)\left(\ominus \widetilde{e}_{1}\right) \oplus K\left(0, \widetilde{e}_{0}, \ominus \widetilde{e}_{1}\right)
$$

if: $\widetilde{e}_{2}<0, \widetilde{e}_{1}>0$,

$$
\ominus 2 \widetilde{e}_{2}=\frac{\sigma}{\sigma s} g\left(0, \widetilde{e}_{0}\right) \oplus \frac{\sigma}{\sigma s} g\left(0, \widetilde{e}_{0}\right) \widetilde{e}_{1} \oplus K\left(0, \widetilde{e}_{0}, \widetilde{e}_{1}\right)
$$

if: $\widetilde{e}_{2}<0, \widetilde{e}_{1}<0$,

$$
\ominus 2 \widetilde{e}_{2}=\frac{\sigma}{\sigma s} g\left(0, \widetilde{e}_{0}\right) \oplus \frac{\sigma}{\sigma s} g\left(0, \widetilde{e}_{0}\right)\left(\ominus \widetilde{e}_{1}\right) \oplus K\left(0, \widetilde{e}_{0}, \ominus \widetilde{e}_{1}\right) \text {. }
$$

So, with comparison (36) and (38), we conclude that

$$
2 \widetilde{e}_{2}=\widetilde{f}^{\prime \prime}(0) \Longrightarrow \widetilde{e}_{2}=\frac{\widetilde{f}^{\prime \prime}(0)}{2 !}
$$

or

$$
\ominus 2 \widetilde{e}_{2}=\widetilde{f}^{\prime \prime}(0) \Longrightarrow \ominus \widetilde{e}_{2}=\frac{\tilde{f}^{\prime \prime}(0)}{2 !} .
$$

By constituting the above procedure, we can easily prove (30) for $m=3,4, \ldots$. 


\section{Numerical Illustrations}

Example 18. Consider the following system of fuzzy linear Volerra integrodifferential equations:

$$
\begin{aligned}
\tilde{x}_{1}^{\prime}(s)= & g_{1}\left(s, \tilde{x}_{1}(s), \tilde{x}_{2}(s)\right) \\
& \ominus \int_{0}^{s} k_{1}\left(s, t, \tilde{x}_{1}(t), \tilde{x}_{2}(t)\right) d t, \\
\tilde{x}_{2}^{\prime}(s)= & g_{2}\left(s, \tilde{x}_{1}(s), \tilde{x}_{2}(s)\right) \\
& \ominus \int_{0}^{s} k_{2}\left(s, t, \tilde{x}_{1}(t), \tilde{x}_{2}(t)\right) d t,
\end{aligned}
$$

with initial conditions

$$
\begin{aligned}
& \tilde{x}_{1}(0)=(1+0.5(r-1), 1-0.5(r-1)), \\
& \tilde{x}_{2}(0)=(-1+0.5(r-1),-1-0.5(r-1)), \\
& g_{1}\left(s, \tilde{x}_{1}(s), \tilde{x}_{2}(s)\right)=1+s+s^{2} \ominus \tilde{x}_{2}(s), \\
& g_{2}\left(s, \tilde{x}_{1}(s), \tilde{x}_{2}(s)\right)=-1-s \oplus \tilde{x}_{1}(s), \\
& k_{1}\left(s, t, \tilde{x}_{1}(t), \tilde{x}_{2}(t)\right)=\tilde{x}_{1}(t) \oplus \tilde{x}_{2}(t), \\
& k_{2}\left(s, t, \tilde{x}_{1}(t), \tilde{x}_{2}(t)\right)=\tilde{x}_{1}(t) \ominus \tilde{x}_{2}(t) .
\end{aligned}
$$

From the initial conditions

$$
\widetilde{e}_{0}=\left[\begin{array}{c}
1+0.5(r-1), 1-0.5(r-1) \\
-1+0.5(r-1),-1-0.5(r-1)
\end{array}\right]^{T} .
$$

Let the solution of (41) be

$$
\begin{aligned}
\tilde{x}_{1}(s) & =\widetilde{e}_{10} \oplus \widetilde{e}_{11} \odot s \\
& =[1+0.5(r-1), 1-0.5(r-1)] \oplus \widetilde{e}_{11} \odot s, \\
\tilde{x}_{2}(s) & =\widetilde{e}_{20} \oplus \widetilde{e}_{21} \odot s \\
& =[-1+0.5(r-1),-1-0.5(r-1)] \oplus \widetilde{e}_{21} \odot s .
\end{aligned}
$$

For obtaining $\widetilde{e}_{11}, \widetilde{e}_{21}$, we substitute (44) into (41); then we will have

$$
\begin{aligned}
& \left(\underline{e}_{11}-2-0.5(r-1)\right) \\
& +\left(-s-s^{2}+\bar{e}_{21} s+(1-r) s-\frac{\bar{e}_{11} s^{2}}{2}-\frac{\underline{e}_{21} s^{2}}{2}\right)=0 \\
& \left(\bar{e}_{11}-2+0.5(r-1)\right) \\
& +\left(-s-s^{2}+\underline{e}_{21} s+(1-r) s-\frac{\underline{e}_{11} s^{2}}{2}-\frac{\bar{e}_{21} s^{2}}{2}\right)=0 \\
& \left(\underline{e}_{21}-0.5(r-1)\right)+\left(3 s-\underline{e}_{11} s+\frac{\bar{e}_{11} s^{2}}{2}+\frac{\underline{e}_{21} s^{2}}{2}\right)=0 \\
& \left(\bar{e}_{21}+0.5(r-1)\right)+\left(3 s-\bar{e}_{11} s+\frac{\underline{e}_{11} s^{2}}{2}+\frac{\bar{e}_{21} s^{2}}{2}\right)=0
\end{aligned}
$$

$$
\begin{aligned}
& \left(\underline{e}_{11}-2-0.5(r-1)\right)+\underline{q}_{11}(s)=0, \\
& \left(\bar{e}_{11}-2+0.5(r-1)\right)+\bar{q}_{11}(s)=0, \\
& \left(\underline{e}_{21}-0.5(r-1)\right)+\underline{q}_{21}(s)=0, \\
& \left(\bar{e}_{21}+0.5(r-1)\right)+\bar{q}_{21}(s)=0,
\end{aligned}
$$

where $\underline{q}_{11}(s), \bar{q}_{11}(s), \underline{q}_{21}(s), \bar{q}_{21}(s)$ are $O(s)$ and by neglecting them, we have

$$
A_{1} \odot \widetilde{e}_{1}=\widetilde{b}_{1}
$$

where

$$
\begin{gathered}
A_{1}=\left[\begin{array}{ll}
1 & 0 \\
0 & 1
\end{array}\right], \\
\widetilde{b}_{1}=\left[\begin{array}{c}
(2+0.5(r-1), 2-0.5(r-1)) \\
(0.5(r-1),-0.5(r-1))
\end{array}\right], \\
\widetilde{e}_{1}=\left[\begin{array}{c}
\left(\underline{e}_{11}, \bar{e}_{11}\right) \\
\left(\underline{e}_{21}, \bar{e}_{21}\right)
\end{array}\right] .
\end{gathered}
$$

So,

$$
\widetilde{e}_{1}=\left[\begin{array}{c}
(2+0.5(r-1), 2-0.5(r-1)) \\
(0.5(r-1),-0.5(r-1))
\end{array}\right] .
$$

And then

$$
\begin{aligned}
\tilde{x}_{1}(s)= & {[1+0.5(r-1), 1-0.5(r-1)] } \\
& \oplus[2+0.5(r-1), 2-0.5(r-1)] \odot s, \\
\tilde{x}_{2}(s)= & {[-1+0.5(r-1),-1-0.5(r-1)] } \\
& \oplus[0.5(r-1),-0.5(r-1)] \odot s .
\end{aligned}
$$

We go to next step. Let

$$
\begin{aligned}
\tilde{x}_{1}(s)= & {[1+0.5(r-1), 1-0.5(r-1)] } \\
& \oplus[2+0.5(r-1), 2-0.5(r-1)] \odot s \oplus \tilde{e}_{12} s^{2}, \\
\tilde{x}_{2}(s)= & {[-1+0.5(r-1),-1-0.5(r-1)] } \\
& \oplus[0.5(r-1),-0.5(r-1)] \odot s \oplus \tilde{e}_{22} s^{2} .
\end{aligned}
$$

Similar to previous step, by substituting (50) into (41), we have

$$
\begin{aligned}
& \left(2 \underline{e}_{12}-1.5(r-1)-1\right) s \\
& \quad+\left(\underline{e}_{22} s^{2}-(r-1) \frac{s^{2}}{2}+\frac{\bar{e}_{12} s^{3}}{3}+\frac{\bar{e}_{22} s^{3}}{3}\right)=0
\end{aligned}
$$




$$
\begin{aligned}
& \left(2 \bar{e}_{12}+1.5(r-1)-1\right) s \\
& \quad+\left(\underline{e}_{22} s^{2}+(r-1) \frac{s^{2}}{2}+\frac{\underline{e}_{12} s^{3}}{3}+\frac{e_{22} s^{3}}{3}\right)=0 \\
& \left(2 \underline{e}_{22}-1.5(r-1)+1\right) s \\
& \quad+\left(s^{2}-\underline{e}_{22} s^{2}+(r-1) \frac{s^{2}}{2}+\frac{\bar{e}_{12} s^{3}}{3}+\frac{\bar{e}_{22} s^{3}}{3}\right)=0 \\
& \left(2 \bar{e}_{22}+1.5(r-1)+1\right) s \\
& \quad+\left(s^{2}+\underline{e}_{22} s^{2}+(r-1) \frac{s^{2}}{2}+\frac{\underline{e}_{12} s^{3}}{3}+\frac{\underline{e}_{22} s^{3}}{3}\right)=0
\end{aligned}
$$

So,

$$
\begin{aligned}
& \left(2 \underline{e}_{12}-1.5(r-1)-1\right) s+\underline{q}_{12}(s)=0, \\
& \left(2 \bar{e}_{12}+1.5(r-1)-1\right) s+\bar{q}_{12}(s)=0, \\
& \left(2 \underline{e}_{22}-1.5(r-1)+1\right) s+\underline{q}_{22}(s)=0, \\
& \left(2 \bar{e}_{22}+1.5(r-1)+1\right) s+\bar{q}_{22}(s)=0 .
\end{aligned}
$$

By neglecting $\underline{q}_{12}(s), \bar{q}_{12}(s), \underline{q}_{22}(s), \bar{q}_{22}(s)$ which are $O\left(s^{2}\right)$ and solve system $A_{2} \odot \widetilde{e}_{2}=\widetilde{b}_{2}$, we obtain

$$
\widetilde{e}_{2}=\left[\begin{array}{l}
\left(\frac{3}{4}(r-1)+\frac{1}{2},-\frac{3}{4}(r-1)+\frac{1}{2}\right) \\
\left(\frac{3}{4}(r-1)-\frac{1}{2},-\frac{3}{4}(r-1)-\frac{1}{2}\right)
\end{array}\right]
$$

and then in a similar way go to next step and we have

$$
\begin{aligned}
& \left(3 \underline{e}_{13}-\frac{5}{4}(r-1)-0.5\right) s^{2}+\underline{q}_{13}(s)=0, \\
& \left(3 \bar{e}_{13}-\frac{5}{4}(r-1)-0.5\right) s^{2}+\bar{q}_{13}(s)=0, \\
& \left(3 \underline{e}_{23}-\frac{5}{4}(r-1)+0.5\right) s^{2}+\underline{q}_{23}(s)=0, \\
& \left(3 \bar{e}_{23}+\frac{5}{4}(r-1)+0.5\right) s^{2}+\bar{q}_{23}(s)=0 .
\end{aligned}
$$

And

$$
\tilde{e}_{3}=\left[\begin{array}{c}
\left(\frac{5}{12}(r-1)+\frac{1}{6},-\frac{5}{12}(r-1)+\frac{1}{6}\right) \\
\left(\frac{5}{12}(r-1)+\frac{1}{6},-\frac{5}{12}(r-1)+\frac{1}{6}\right)
\end{array}\right] .
$$

Example 19. As second example we consider the following nonlinear fuzzy integrodifferential equation:

$$
\begin{aligned}
& \tilde{x}^{\prime}(s)=(1,0.1,0.4) \oplus \int_{0}^{s} \tilde{x}(t) \odot \tilde{x}^{\prime}(t) d t, \\
& \tilde{x}(0)=(0,0.2,0.6) .
\end{aligned}
$$

Typically, we use the power series method for obtaining the solution of problem. From the initial condition, $\widetilde{e}(0)=$ $(0,0.2,0.6)$, let the solution of $(56)$ be the form

$$
\tilde{x}(s)=\widetilde{e}_{0} \oplus \widetilde{e}_{1} \odot s=(0,0.2,0.6) \oplus \widetilde{e}_{1} \odot s .
$$

For obtaining $\widetilde{e}_{1}=(m, \alpha, \beta)$, we substitute (57) into (56); we will have

$$
(m, \alpha, \beta)=\left(1+\frac{m s^{2}}{2}, 0.1+\frac{\alpha s^{2}}{2}+0.2 s, 0.4+\frac{\beta s^{2}}{2}+0.3 s\right)
$$

or

$$
(m, \alpha, \beta)=\left(1+q_{1}(s), 0.1+q_{2}(s), 0.4+q_{3}(s)\right) .
$$

By neglecting $q_{1}, q_{2}, q_{3}$ which are $O(s)$, we obtain $\widetilde{e}_{1}=$ $(1,0.1,0.4)$ and then

$$
\tilde{x}(s)=(0,0.2,0.6) \oplus(1,0.1,0.4) \odot s .
$$

For the next step, we assume that

$$
\tilde{x}(s)=\widetilde{e}_{0} \oplus \tilde{e}_{1} \odot s \oplus \tilde{e}_{2} \odot s^{2} .
$$

By substituting (61) into (56), we have

$$
\begin{aligned}
(1 & +2 m s, 0.1+\alpha s, 0.4+\beta s) \\
& =\left(1+\frac{s^{2}}{2}, 0.1+0.2 s+q_{1}(s), 0.4+0.6 s+q_{2}(s)\right) .
\end{aligned}
$$

From above relation and by neglecting $s^{2} / 2, q_{1}(s), q_{2}(s)$, we have $\widetilde{e_{2}}=(0,0.1,0.3)$.

By repeating this method, we can compute more coefficients of the solution.

Example 20. Consider the following nonlinear fuzzy integrodifferential equation:

$$
\begin{aligned}
& \tilde{x}^{\prime}(s)=e^{s}-\frac{1}{3} e^{3 s}+\left(\frac{1}{3}, 0.1,0.3\right) \oplus \int_{0}^{s} \widetilde{x}^{3}(t) d t, \\
& \tilde{x}(0)=(1,0.4,0.4) .
\end{aligned}
$$

Again, we use the power series method for obtaining the solution of the problem. From the initial condition, $\widetilde{e}_{0}=$ $(1,0.4,0.4)$. Assume that the solution of $(63)$ is the form

$$
\tilde{x}(s)=\tilde{e}_{0} \oplus \tilde{e}_{1} \odot s=(1,0.4,0.4) \oplus \tilde{e}_{1} \odot s .
$$

By substituting (64) into (63), we obtain

$$
(m, \alpha, \beta)=\left(1+q_{1}(s), 0.4+q_{2}(s), 0.4+q_{3}(s)\right) .
$$

By neglecting $q_{1}(s), q_{2}(s), q_{3}(s)$, we obtain $\widetilde{e}_{1}=(1,0.4,0.4)$ and then

$$
\tilde{x}(s)=(1,0.4,0.4) \oplus(1,0.4,0.4) \odot s .
$$

For the next step, we assume that

$$
\widetilde{x}(s)=(1,0.4,0.4) \oplus(1,0.4,0.4) s \oplus \widetilde{e}_{2} \odot s^{2} .
$$


And by substituting it into (63), we have

$$
\begin{aligned}
(1 & +2 m s, 0.4+2 \alpha s, 0.4+2 \beta s) \\
& =\left(1+s+q_{1}(s), 0.4+0.6 s+q_{2}(s), 0.4+0.6 s+q_{3}(s)\right) .
\end{aligned}
$$

From the above relation and by neglecting $q_{1}(s), q_{2}(s), q_{3}(s)$, we have

$$
\widetilde{e}_{2}=\left(\frac{1}{2}, 0.3,0.3\right)
$$

By continuing this procedure, more coefficients of the solution can be computed.

\section{Conclusion}

In summary, this study has exploited power series to find a numerical solution for linear as well as nonlinear fuzzy Volterra integrodifferential equations. In effect, using power series can provide an approximate solution for the mentioned integral equations. Since there are challenging issues to solve the nonlinear integrodifferential equations, the presented method can be simply applied to find an appropriate solution for this kind of equations that is regarded as a considerable benefit of this method undoubtedly.

\section{Conflict of Interests}

The authors declare that there is no conflict of interests regarding the publication of this paper.

\section{References}

[1] S. S. L. Chang and L. A. Zadeh, "On fuzzy mapping and control," IEEE Transactions on Systems, Man, and Cybernetics, vol. 2, pp. 30-34, 1972.

[2] L. A. Zadeh, "The concept of a linguistic variable and its application to approximate reasoning," Information Sciences, vol. 8, no. 3, pp. 199-249, 1975.

[3] M. Mizumoto and K. Tanaka, "The four operations of arithmetic on fuzzy numbers," Systems Computers Controls, vol. 7, no. 5, pp. 73-81, 1976.

[4] D. Dubois and H. Prade, "Operations on fuzzy numbers," International Journal of Systems Science, vol. 9, no. 6, pp. 613626, 1978.

[5] S. Seikkala, "On the fuzzy initial value problem," Fuzzy Sets and Systems, vol. 24, no. 3, pp. 319-330, 1987.

[6] R. Goetschel Jr. and W. Voxman, "Elementary fuzzy calculus," Fuzzy Sets and Systems, vol. 18, no. 1, pp. 31-43, 1986.

[7] R. Alikhani, F. Bahrami, and A. Jabbari, "Existence of global solutions to nonlinear fuzzy Volterra integro-differential equations," Nonlinear Analysis: Theory, Methods \& Applications, vol. 75, no. 4, pp. 1810-1821, 2012.

[8] T. Allahviranloo, M. Khezerloo, O. Sedaghatfar, and S. Salahshour, "Toward the existence and uniqueness of solutions of second-order fuzzy volterra integro-differential equations with fuzzy kernel," Neural Computing and Applications, vol. 22, no. 1, pp. 133-141, 2013.
[9] S. Hajighasemi, T. Allahviranloo, M. Khezerloo, M. Khorasany, and S. Salahshour, "Existence and uniqueness of solutions of fuzzy Volterra integro-differential equations," in Information Processing and Management of Uncertainty in Knowledge-Based Systems, vol. 81 of Communications in Computer and Information Science, part 2, pp. 491-500, 2010.

[10] B. Bede and L. Stefanini, "Generalized differentiability of fuzzyvalued functions," Fuzzy Sets and Systems, vol. 230, pp. 119-141, 2013.

[11] S. Salahshour and S. Abbasbandy, "A comment on "Global solutions for nonlinear fuzzy fractional integral and integrodifferential equations'”' Communications in Nonlinear Science and Numerical Simulation, vol. 19, no. 5, pp. 1256-1258, 2014.

[12] T. Allahviranloo and S. Hashemzehi, "The homotopy perturbation method for fuzzy Fredholm integral equations," Journal of Applied Mathematics, Islamic Azad University of Lahijan, vol. 19, pp. 1-13, 2008.

[13] M. Gachpazan, "Numerical scheme to solve integro-differential equations system," Journal of Advanced Research in Scientific Computing, vol. 1, no. 1, pp. 11-21, 2009.

[14] B. Bede and S. G. Gal, "Generalizations of the differentiability of fuzzy-number-valued functions with applications to fuzzy differential equations," Fuzzy Sets and Systems, vol. 151, no. 3, pp. 581-599, 2005.

[15] L. A. Zadeh, "Fuzzy sets," Information and Computation, vol. 8, pp. 338-353, 1965.

[16] H. J. Zimmermann, Fuzzy Sets Theory and Its Applications, Kluwer, Dordrecht, The Netherlands, 1991.

[17] D. Dubois and H. Prade, Fuzzy Sets and Systems, Academic Press, London, UK, 1980.

[18] H. T. Nguyen, "A note on the extension principle for fuzzy sets," Journal of Mathematical Analysis and Applications, vol. 64, no. 2, pp. 369-380, 1978.

[19] M. L. Puri and D. A. Ralescu, "Fuzzy random variables," Journal of Mathematical Analysis and Applications, vol. 114, no. 2, pp. 409-422, 1986.

[20] M. Friedman, M. Ma, and A. Kandel, "Numerical solutions of fuzzy differential and integral equations," Fuzzy Sets and Systems, vol. 106, no. 1, pp. 35-48, 1999.

[21] Y. Chalco-Cano and H. Román-Flores, "On new solutions of fuzzy differential equations," Chaos, Solitons and Fractals, vol. 38, no. 1, pp. 112-119, 2006.

[22] T. Allahviranloo, N. Mikaeilvand, N. A. Kiani, and R. M. Shabestari, "Signed decomposition of fully fuzzy linear systems," Applications and Applied Mathematics, vol. 3, no. 1, pp. 77-88, 2008. 


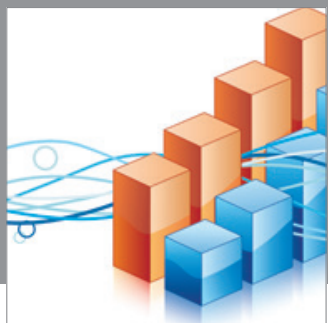

Advances in

Operations Research

mansans

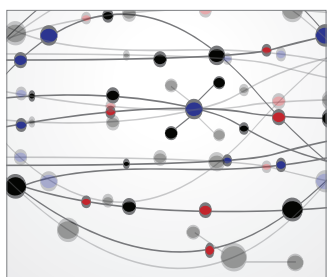

The Scientific World Journal
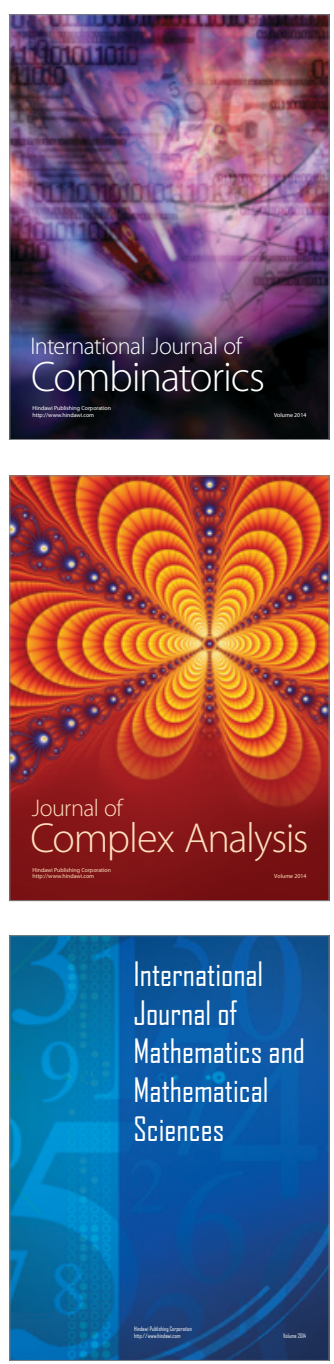
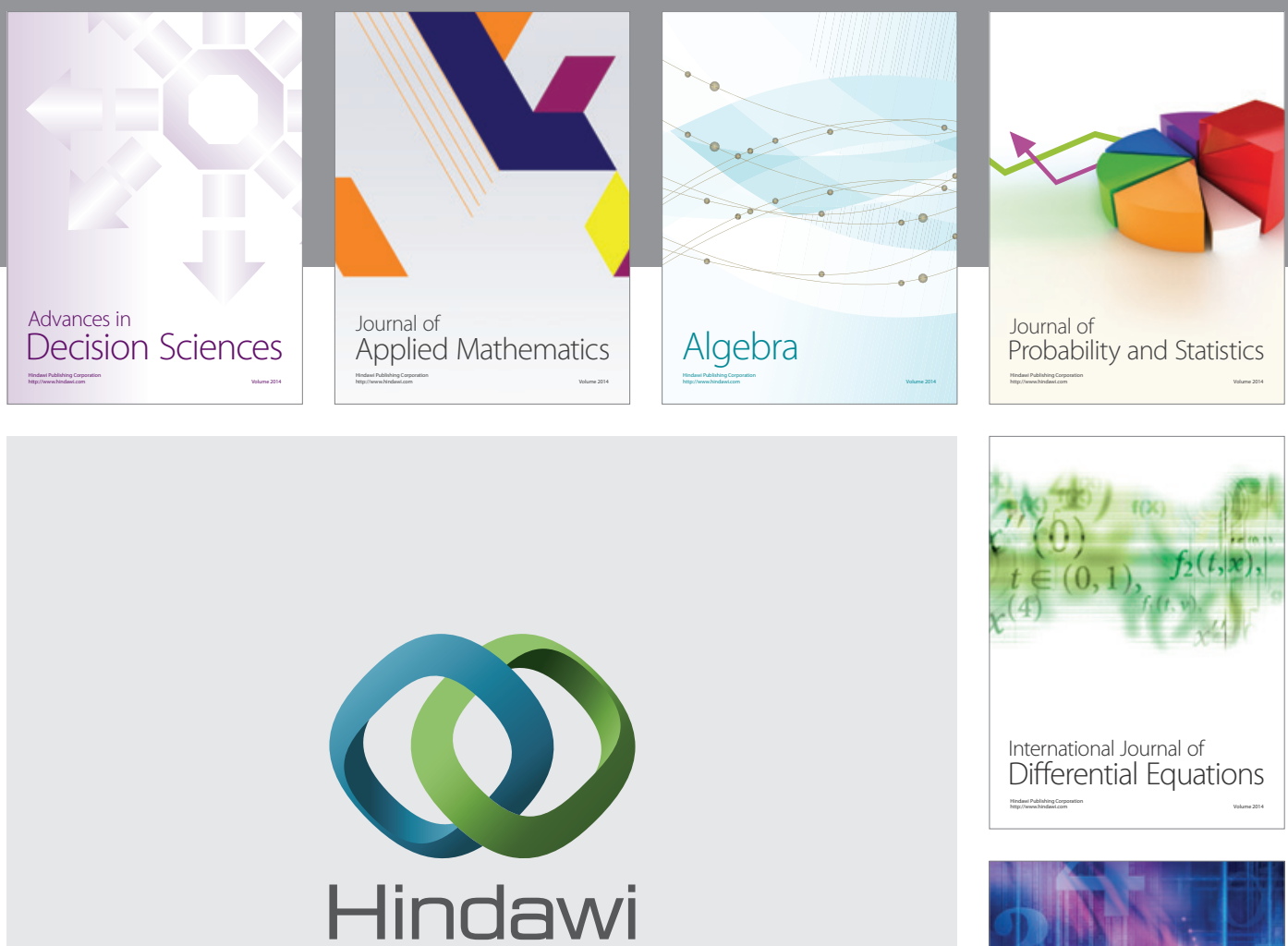

Submit your manuscripts at http://www.hindawi.com
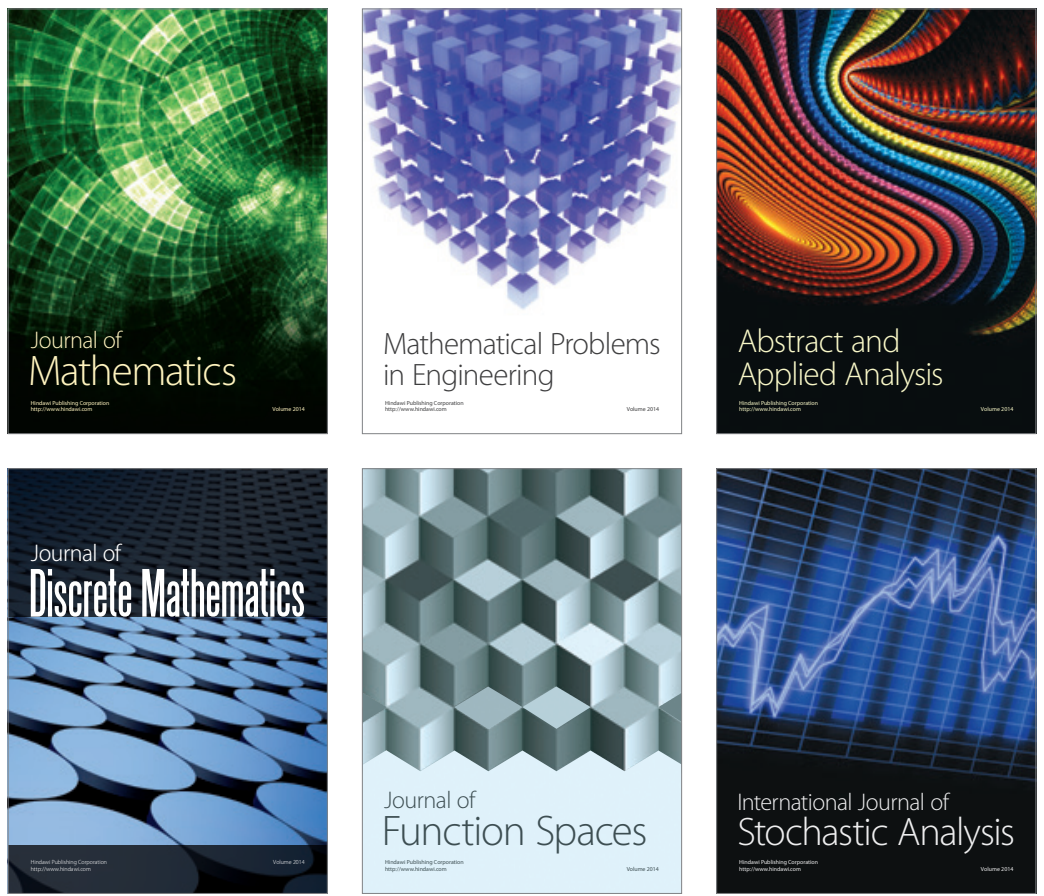

Journal of

Function Spaces

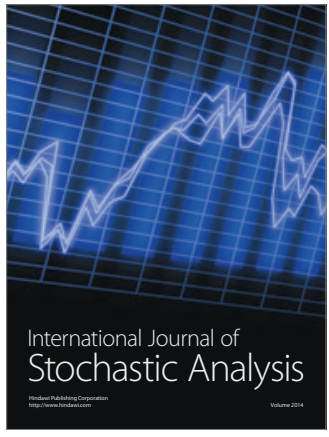

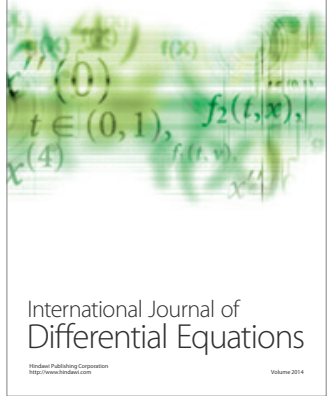
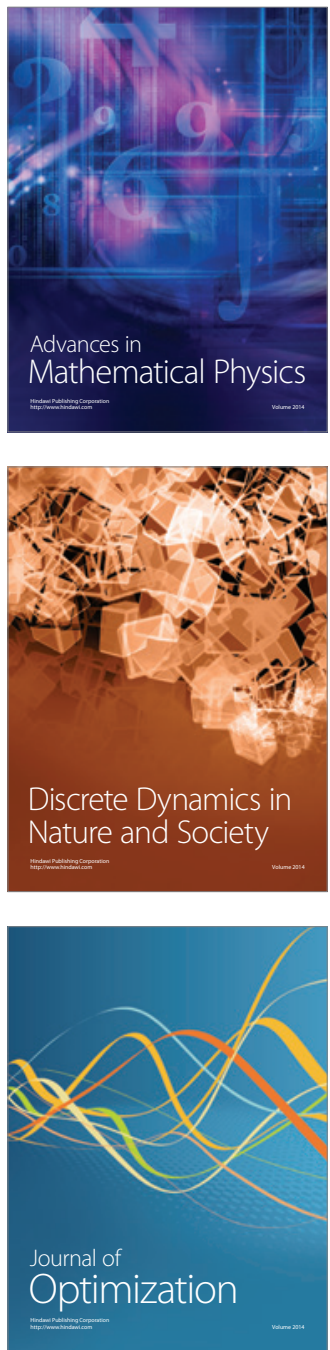